\title{
Interpreting and addressing suboptimal immune responses after COVID-19 vaccination in solid-organ transplant recipients
}

\author{
Peter G. Stock, ${ }^{1}$ Timothy J. Henrich, ${ }^{2}$ Dorry L. Segev, ${ }^{3}$ and William A. Werbel ${ }^{4}$ \\ 'Department of Surgery and ${ }^{2}$ Department of Medicine, UCSF, San Francisco, California, USA. ${ }^{3}$ Department of Surgery and ${ }^{4}$ Department of Medicine, Johns Hopkins University School of Medicine, Baltimore, \\ Maryland, USA.
}

Transplant recipients were excluded from the initial clinical trials determining safety and efficacy of the landmark COVID-19 vaccines. Further, there is increasing evidence that immunosuppressed transplant recipients have a blunted antibody response to COVID-19 vaccination. In a concerning report by Sattler et al. in this issue of the $J \mathrm{Cl}$, kidney transplant recipients not only lacked a humoral response following two doses of Pfizer BNT162b2, but also displayed substantial impairment of the cellular response to SARS-CoV-2 antigens. This Commentary addresses potential strategies for transplant providers to evaluate and augment vaccine immunogenicity given the likelihood that COVID-19 will remain a worldwide threat to the health of transplant recipients.

\section{Transplant recipients may} remain at risk for COVID-19

The COVID-19 pandemic has challenged health care providers in the transplant community to provide management strategies for immunosuppressed transplant recipients in the absence of conventional evidence-based data or peer-reviewed literature that traditionally shape clinical practice. The lack of evidence regarding the immunogenicity of COVID-19 vaccination is particularly problematic in transplant recipients, who were excluded from the clinical trials determining the safety and efficacy of the landmark vaccines, yet have suffered severe COVID-19 burden, with a case fatality ratio that spans $10 \%$ to $30 \%(1-3)$.

Although early data indicate that the vaccines are safe and are thus strongly recommended for all transplant recipients, multiple reports indicate dampened antibody responses after two doses of mRNA vaccines $(4,5)$. It is not surprising that humoral vaccine response is reduced in transplant recipients on chronic immunosuppressives (6), since this is well described following standard influenza vaccination $(7,8)$. It is, however, disappointing that nearly $50 \%$ of transplant recipients do not develop anti-spike seroresponse to vaccination, given the impressive immunogenicity of these vaccines in the general population. These highly publicized findings, plus growing reports of symptomatic breakthrough cases after vaccination in transplant recipients (9), raise concern that a marked proportion of transplant recipients may remain at risk for COVID-19.

Delated Article: https://doi.org/10.1172/JCI150175

Conflict of interest: TJH receives grant support from Gilead Biosciences, Bristol Meyer Squibb, and Merck \& Co. DLS has served as a consultant to and received honoraria for speaking from Sanofi, Novartis, CSL Behring, Jazz Pharmaceuticals, Veloxis, Mallinckrodt, and Thermo Fisher Scientific.

Copyright: () 2021, American Society for Clinical Investigation.

Reference information: J Clin Invest. 2021;131(14):e151178. https://doi.org/10.1172/JCI151178.

Clinicians and scientists have voiced hope that cellular responses, as implicated following influenza vaccination (10), might protect from serious disease. Published data on $\mathrm{T}$ cell responses to COVID-19 vaccination in transplant recipients, although important, have been limited (e.g., IFN- $\gamma$ release assays; refs. 11, 12). The report by Sattler et al. in this issue of the JCI (13) uses in-depth immunophenotyping to report further concerning data that demonstrate not only lack of humoral response, but notable impairment of cellular response following two doses of Pfizer BNT162b2 (tozinameran; Comirnaty) in a small cohort of kidney transplant recipients. Although the humoral response after two doses of mRNA vaccination in transplant recipients was $54 \%$ in the Boyarsky et al. series (5), it was a dismal $2.6 \%$ percent in the series of Sattler et al. studying a population of predominately middle-aged, White kidney transplant recipients on triple immunosuppression (calcineurin inhibitors [CNI], antimetabolites, and corticosteroids). Of equal importance, these participants demonstrated very diminished cellular response to SARSCoV-2 antigens following completion of the mRNA vaccine series, which was particularly evident in the dearth of spike-specific $\mathrm{CD}^{+} \mathrm{T}$ cell responses. Thus, it is challenging to assume that cellular responses will be a deus ex machina for the majority of transplant patients who do not develop evidence of humoral response to vaccination.

\section{Recommendations and guidelines}

Transplant recipients are anxiously, and appropriately, reaching out to transplant centers to provide recommendations 
mRNA booster vaccination

Pfizer BioNTech 162b2

Moderna mRNA-1273
Pros

Cons

Hypersensitivity

(rare)

(especially humoral)

Reassuring safety

protocol

Unknowns

Alloimmune stimulation,

e.g., donor-specific antibody

Optimal antigen dosing

Tolerance/anergy with

repeated administration

\section{Adenovirus \\ (heterologous) vaccination \\ Johnson \& Johnson/Janssen (Ad26.COV2.S) Oxford-AstraZeneca (ChAdOx1/AZD1222) \\ Sputnik V (Gam-Covid-Vac) \\ CanSinoBIO (Ad5-nCov)}

Thrombosis (rare) Increased reactogenicity with heterologous prime-boost
Alloimmune stimulation, e.g., donor-specific antibody Improved adaptive cellular responses Preexisting adenovirusneutralizing antibodies

\section{Virus-like particle (heterologous) vaccination Novavax (NVX-Cov2373)}

Immunogenic
More extensive track record

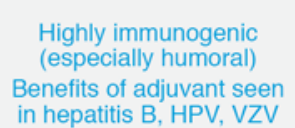

Highly immunogenic Benefits of adjuvant seen in hepatitis B, HPV, VZV
Less published

data
Alloimmune stimulation

(adjuvant)

$$
\begin{aligned}
& \text { Tightly controlled, } \\
& \text { reversible intervention } \\
& \text { Extensive provider } \\
& \text { experience }
\end{aligned}
$$

\section{Rejection risk in \\ immunostimulatory environment}

Mycophenolate derivative

Calcineurin inhibitors

Corticosteroids

mTOR inhibitors

Figure 1. Potential strategies for improving vaccine immunogenicity for transplant recipients.

regarding the necessity of testing for an immune response after vaccination as well as interpretation of results and strategies for addressing a blunted or absent humoral response following the second dose (14). The most recent CDC and FDA guidelines do not recommend serologic testing following COVID-19 vaccination $(15,16)$. This recommendation is partly based on the impressive impact of the mRNA vaccines in the prevention of severe illness or death from COVID-19 in the general population as well as the uncertain impact of a blunted anti-spike antibody response using the myriad of commercially available tests with varying operating characteristics. For similar reasons, the American Society of Transplantation (AST) does not recommend serologic testing in transplant recipients following COVID-19 vaccination (17). Nonetheless, these data from Sattler showing impairment of cellular responses and dovetailing with previously reported lack of humoral responses highlight the importance of evaluating vaccine-associated immune response in immunosuppressed transplant recipients.

At this point in time, although commercial serologic testing is limited to quantifying antibody levels rather than formal neutralization capacity, FDA emergency use authorized assays that target the S1 subunit of the spike protein have merit in assessing humoral response to vaccination, given the strong correlation with neutralizing antibody that in turn is associated with vaccine effectiveness (18). In addition to measuring circulating proteins that can block viral entry, high levels of antibody also serve as a surrogate for B cell competency and response to vaccine antigen. As such, monitoring of vaccine responses in transplant recipients with an appropriate anti-spike assay and interpreted by an informed transplant provider may guide clinical decision making and pointed counseling. Although we concur with the AST that the ramifications of a blunted antibody response are not fully known, it is logical that transplant recipients with a minimal antibody response should receive strong counseling on behavior modification as advised by the CDC as, effectively, unvaccinated people who should continue to practice social distancing and masking, particularly in crowds and among unvaccinated persons (19). Similarly, the finding of low antibody levels in an immunocompromised person, despite vaccination, may be further impetus for cohabitants and coworkers to undergo vaccination to provide the local and community protection that the vaccines themselves may not yet offer for transplant recipients.

\section{Strategies for improving vaccine immunogenicity}

The cellular response data described in the paper by Sattler et al. may be helpful in understanding the mechanisms of impaired vaccine immune response in immunosuppressed patients as well as potential strategies for altering immunosuppressive medications during the vaccination window or offering a different platform for a subsequent booster vaccination However, cellular assays to measure vaccine responses are not commercially available. Viral-specific helper $\mathrm{CD} 4^{+} \mathrm{T}$ cell responses have been shown to correlate with antibody levels and neutralization titers (20-22). Importantly, cutoffs for protective $\mathrm{CD}^{+}$ or cytolytic $\mathrm{CD}^{+} \mathrm{T}$ cell responses have not been defined in any population. Cucchiari et al. (23) recently demonstrated that SARS-CoV-2-specific T cell responses can be observed in up to $50 \%$ of kidney transplant recipients who do not develop antibodies following vaccination. Unlike antibody responses, however, it is not yet known whether these $\mathrm{T}$ cell responses correlate with protection versus SARS-CoV-2 infection or attenuate disease severity, particularly in persons taking immunosuppressives explicitly tailored to blunt $\mathrm{T}$ cell activity (i.e., inhibit alloimmunity). 
What are potential strategies for improving vaccine immunogenicity given the likelihood that COVID-19 will remain a world-wide threat to the health of transplant recipients (Figure 1 and ref. 24)? Alternative vaccination regimens, including additional doses of mRNA vaccines, are currently being assessed in clinical trials, including a Canadian trial of a third booster dose of Moderna COVID-19 vaccine in transplant recipients (25). The French Health ministry now routinely recommends a third dose of mRNA vaccines for recipients of bone marrow and solid organ transplants as well other immunosuppressed populations (26). There are also anecdotal reports (14) of third doses in the United States, including an antibody response to an adenoviral vectored vaccine after a failed response to two doses of mRNA vaccine in a transplant recipient (i.e., heterologous boosting). Indeed, there is some evidence that the Oxford-AstraZeneca adenovirus vaccine ChAdOx1 stimulates a more potent cellular response than BNT162b2 mRNA vaccine in elderly persons (27). Based on the report by Sattler et al., it will be important to prospectively study both the humoral and cellular responses using any alternative dosing strategies as well as associated modulation of immunosuppression regimens. In that way, approaches that are optimal in improving the broad impairment in effector cytokine production, memory differentiation, and spike-specific CD8 ${ }^{+} \mathrm{T}$ cell responses as noted in this study can be adopted into vaccination strategies.

Modulation of immunosuppressives is another potential strategy. The lack of an antibody response following completion of the mRNA vaccines has been strongly correlated with the utilization of the antiproliferative agent mycophenolic mofetil (MMF) in the immunosuppressive regimens of transplant recipients $(5,6)$. This has also been observed in influenza vaccination (8). All of the recipients in the Sattler study were maintained on immunosuppressive regimens, including a CNI and an MMF; hence, the impact of the antiproliferative agent could not be determined. One potential strategy for enhancing the immune response in transplant recipients could be to minimize or potentially hold the antiproliferative agent at the time of a vaccine booster in patients with a failed vaccinemediated antibody response. Although not recommended by transplant societies at this time, this approach has been explicitly recommended for persons taking antiproliferative agents for control of autoimmune diseases (28). This strategy must be nuanced and individualized, as for example, recipients with recent transplantation and/or who are highly allosensitized could be at unacceptably high risk for rejection or other alloimmune events. Fortunately, to date, there is no strong evidence to support enhancement of the alloimmune response by COVID-19 vaccination in transplant recipients (29). For recipients that are deemed low risk by their transplant provider for developing an alloimmune complication in the context of antiproliferative agent minimization, for example, long-standing liver transplant recipients, the ability to modulate immunosuppression at the time of the vaccine booster may be a reasonable approach after individualized discussion of the risks and benefits.

In order to deliver optimal vaccine responses for our transplant recipients, it will be important to conduct careful, monitored clinical trials of vaccine boosters as well as modulation of immunosuppression regimens. Nonetheless, many transplant recipients do not have access to such clinical trials and deserve information in real time, particularly in regions suffering unchecked viral spread and in advance of anticipated seasonal coronavirus disease spikes. As with all management strategies for transplantation during the COVID-19 pandemic, any of the outlined approaches will have to be made in a relative data vacuum alongside individualized discussion between clinicians and transplant recipients, carefully integrating rapidly evolving observational and clinical trial results into that vague but important notion of clinical judgment. In the interim, appropriate testing for humoral responses following vaccination in transplant recipients would provide data for encouraging and reinforcing safe behavior and would identify the highest risk individuals who may benefit from these exploratory, but crucial interventions.

\section{Acknowledgments}

PGS is supported by NIH U01AI118594 and California Institute of Regenerative Medicine (CIRM) CLIN2-11437. WAW is supported by an AST Research Network/Sanofi Fellowship Clinical Science research grant.
Address correspondence to: Peter G. Stock, University of California, San Francisco, 505 Parnassus Avenue, M-884, Box 0780, San Francisco, California 94143-0780, USA. Phone: 415.353.8617; Email: Peter.Stock@ ucsf.edu.

1. Heldman MR, et al. Covid-19 in hospitalized lung and non-lung solid organ transplant recipients: a comparative analysis from a multicenter study [published online May 19, 2021]. Am J Transplant. https://doi.org/10.1111/ajt.16692.

2. Molnar MZ, et al. Outcomes of critically ill solid organ transplant patients with COVID19 in the United States. Am J Transplant. 2020;20(11):3061-3071.

3. Raja MA, et al. COVID-19 in solid organ transplant recipients: A systematic review and meta-analysis of current literature. Transplant Rev (Orlando). 2021;35(1):100588.

4. Grupper A, et al. Reduced humoral response to mRNA SARS-CoV-2 BNT162b2 vaccine in kidney transplant recipients without prior exposure to the virus [published online April 18, 2021]. Am J Transplant. https://doi.org/10.1111/ajt.16615.

5. Boyarsky BJ, et al. Antibody response to twodose SARS-CoV-2 messenger RNA vaccine series in solid organ transplant recipients. JAMA. 2021;325(21):2204-2206.

6. Grupper A, Katchman H. Reduced humoral response to mRNA SARS-Cov-2 BNT162b2 vaccine in kidney transplant recipients without prior exposure to the virus: not alarming, but should be taken gravely [published online June 3, 2021]. Am J Transplant. https://doi.org/10.1111/ajt.16710.

7. Birdwell KA, et al. Decreased antibody response to influenza vaccination in kidney transplant recipients: a prospective cohort study. Am J Kidney Dis. 2009;54(1):112-121.

8. Kumar D, et al. Randomized controlled trial of adjuvanted versus nonadjuvanted influenza vaccine in kidney transplant recipients. Transplantation. 2016;100(3):662-669.

9. Ali NM, et al. Development of COVID-19 infection in transplant recipients after SARS-CoV-2 vaccination [published online May 26, 2021]. Transplantation. https://doi.org/10.1097/ tp.0000000000003836.

10. L'Huillier AG, et al. Cell-mediated immune responses after influenza vaccination of solid organ transplant recipients: secondary outcomes analyses of a randomized controlled trial. J Infect Dis. 2020;221(1):53-62.

11. Miele $\mathrm{M}$, et al. Impaired anti-SARS-CoV-2 humoral and cellular immune response induced by Pfizer-BioNTech BNT162b2 mRNA vaccine in solid organ transplanted patients [published online May 31, 2021]. Am J Transplant. https:// doi.org/10.1111/ajt.16702.

12. Havlin J, et al. Immunogenicity of BNT162b2 mRNA COVID-19 vaccine and SARS-CoV-2 infection in lung transplant recipients [published online May 21, 2021]. J Heart Lung Transplant. https://doi.org/10.1016/j.healun.2021.05.004.

13. Sattler A, et al. Impaired humoral and cellular immunity after SARS-CoV-2 BNT162b2 (tozinameran) prime-boost vaccination in 
kidney transplant recipients. J Clin Invest. 2021;131(14):150175.

14. Barrett, J. Immunocompromised people turn to Covid-19 vaccine booster shots. Wall Street Journal. June 2, 2021. Accessed June 18, 2021. https://www.wsj.com/articles/immunocompromised-people-turn-to-covid-19-vaccine-booster-shots-11622626200.

15. Food and Drug Administration. EUA Authorized Serology Test Performance. https://www. fda.gov/medical-devices/coronavirus-disease-2019-covid-19-emergency-use-authorizations-medical-devices/eua-authorized-serology-test-performance. Updated May 25, 2021. Accessed June 18, 2021.

16. Centers for Disease Control and Prevention. Interim Clinical Considerations for Use of COVID-19 Vaccines Currently Authorized in the United States. https://www.cdc.gov/vaccines/ covid-19/clinical-considerations/covid-19-vaccines-us.html. Updated June 1, 2021. Accessed June 18, 2021.

17. American Society of Transplantaiton. Statement on COVID-19 Vaccination in Solid Organ Transplant Recipients. https://www.myast. org/statement-covid-19-vaccination-solid-organ-transplant-recipients. Updated June 2, 2021. Accessed June 18, 2021.
18. Khoury DS, et al. Neutralizing antibody levels are highly predictive of immune protection from symptomatic SARS-CoV-2 infection [published online May 17,2021]. Nat Med. https://doi. org/10.1038/s41591-021-01377-8.

19. Centers for Disease Control and Prevention. Interim Public Health Recommendations for Fully Vaccinated People. https://www.cdc.gov/ coronavirus/2019-ncov/vaccines/fully-vaccinated-guidance.html. Updated May 28, 2021. Accessed May 30, 2021.

20. Ogbe A, et al. T cell assays differentiate clinical and subclinical SARS-CoV-2 infections from cross-reactive antiviral responses. Nat Commun. 2021;12(1):2055.

21. Dan JM, et al. Immunological memory to SARSCoV-2 assessed for up to 8 months after infection. Science. 2021;371(6529):eabf4063.

22. Sette A, Crotty S. Adaptive immunity to SARSCoV-2 and COVID-19. Cell. 2021;184(4):861-880.

23. Cucchiari D, et al. Cellular and humoral response after mRNA-1273 SARS-CoV-2 vaccine in kidney transplant recipients [published online May 26, 2021]. Am J Transplant. https://doi. org/10.1111/ajt.16701.

24. Klasse PJ, et al. Immunogenicity of clinically relevant SARS-CoV-2 vaccines in nonhuman primates and humans. Sci Adv. 2021;7(12):eabe8065.

25. NIH. Third Dose of Moderna COVID-19 Vaccine in Transplant Recipients. https://clinicaltrials. gov/ct2/show/NCT04885907. Updated June 7, 2021. Accessed June 18, 2021.

26. French Republic. Precisions sur la Vaccination COVID-19: Modalites d'Administration des Rappels et Vaccination des Personnes Immunodeprimes et de Leurs Proches. https://solidarites-sante.gouv.fr/IMG/pdf/dgs_urgent_52_precisions_sur_la_vaccination_imd.pdf. Updated May 5, 2021. Accessed June 18, 2021.

27. Parry HM, et al. Single vaccination with BNT162b2 or ChAdOx1 in older people induces equivalent antibody generation but enhanced cellular responses after ChAdOx1 [preprint]. http://dx.doi.org/10.2139/ssrn.3825573. Posted on SSRN April 13, 2021

28. American College of Rheumatology. COVID19 Guidance. https://www.rheumatology.org/ Practice-Quality/Clinical-Support/COVID19-Guidance. Updated June 15, 2021. Accessed June 18, 2021.

29. Ou MT, et al. Safety and reactogenicity of 2 doses of SARS-CoV-2 vaccination in solid organ transplant recipients [published online April 9, 2021]. Transplantation. https://doi.org/10.1097/ tp.0000000000003780. 